\title{
Neoneurogenesis in Squamous Cell Carcinoma of Tongue: A New Mechanism for Its Development
}

\author{
Zhenqi Xu1 ${ }^{*}$, Jianwei Shang ${ }^{2}$ \\ ${ }^{1}$ Department of Oral and Maxillofacial Surgery, Tianjin Stomatological Hospital, Tianjin, China \\ ${ }^{2}$ Department of Oral Pathology, Tianjin Stomatological Hospital, Tianjin, China \\ Email: *xuzhenqi2001@163.com
}

How to cite this paper: Xu, Z.Q. and Shang, J.W. (2019) Neoneurogenesis in Squamous Cell Carcinoma of Tongue: A New Mechanism for Its Development. Open Journal of Stomatology, 9, 102-107. https://doi.org/10.4236/ojst.2019.95011

Received: April 2, 2019

Accepted: May 10, 2019

Published: May 13, 2019

Copyright () 2019 by author(s) and Scientific Research Publishing Inc. This work is licensed under the Creative Commons Attribution International License (CC BY 4.0).

http://creativecommons.org/licenses/by/4.0/

\section{(c) () Open Access}

\begin{abstract}
Objectives: To explore the role of the growth of new nerves (neo-neurogenesis) in the tumorogenesis of squamous cell carcinoma of tongue. Materials and Methods: 10 formalin-fixed specimens were gained from patients diagnosed with tongue squamous cell carcinoma. Animal models were made by subcutaneous injection in the dorsal midline with Tca- 8113 cell line. Mice were sacrificed 2, 4, 6 weeks after cell injection, and tumor tissues were fixed in $4 \%$ paraformaldehyde, embedded in paraffin, sectioned. Detection of neo-neurogenesis was stained by Neurofilament-L antibody (NF-L) using immunohistochemistry method (IHC) in biopsy from both human body and animal model. Results: IHC staining of NF-L is positive in all 10 paraffins of tongue squamous cell carcinoma sections which suggest that newly formed nerves are observed in tumor microenvironment. NF-L staining is also positive in the paraffins from animal models indicating that the tongue cancer recruits newly formed nerves in its tumorogenesis. Conclusions: Tumor neo-neurogenesis may play an important role in the pathogenesis and development of tongue cancer. From a therapeutic perspective, further studies on the topic may provide new clinical opportunity.
\end{abstract}

\section{Keywords}

Neoneurogenesis, Squamous Cell Carcinoma, Tongue, Neurofilament-L, Immunohistochemistry

\section{Introduction}

Squamous cell carcinoma (SCC) of the tongue is the most common malignancy in the oral cavity with an incidence varying from 1.1 to $9.4 / 100,000 /$ year [1] 
Despite current advances in diagnosis and management, the tongue cancer remains a difficult region to assess and a 5 -year survival remains poor at $62.7 \%$ in the USA and below $50 \%$ in other countries [2].

The exact pathogenesis of tongue cancer has not been clearly uncovered. Recently, few studies have revealed that infiltration of the tumor microenvironment by nerves, termed tumor neoneurogenesis plays an important role in cancer progression and dissemination [3] [4] [5].

In the present study, we investigated whether tumor neoneurogenesis could be observed in SCC of the tongue, and an animal study was designed to explore the role of the growth of new nerves in the tumorogenesis of SCC.

\section{Materials and Methods}

1) Biopsy from patients

10 preexisting human formalin-fixed paraffin embedded tissue histologically confirmed as tongue squamous cell carcinoma were chosen.

2) Cell culture

Tca-8113 cell line was kindly donated by Dr. Wu Debao from Tianjin Medical University and maintained in DMEM supplemented with $10 \%$ heat-inactivated FBS, $100 \mathrm{U} / \mathrm{ml}$ penicillin, $100 \mu \mathrm{g} / \mathrm{ml}$ streptomycin (GIBCO, Grand Island, NY, USA) at $37^{\circ} \mathrm{C}$ in a humidied atmosphere containing $95 \%$ air and $5 \% \mathrm{CO}_{2}$.

3) Murine Model

Fifteen 4 - 6 weeks old female Balb/c nude mice ( $n u / n u$ ), purchased from Charles River laboratories. On the day at tumor implantation, the cell suspension was achieved at a final density of $1.0 \times 10^{7}$ cells $/ \mathrm{ml}$ in chilled PBS. Each mouse received $100 \mu \mathrm{l}$ of cell suspension (namely $1.0 \times 10^{6} \mathrm{Tca}-8113$ cells) by subcutaneous injection in the dorsal midline.

2, 4, 6 weeks after cell injection, the animals were sacrificed under deep anesthesia for collection of tumor tissues (five in each group). All animal experiments were approved by the Animal Care \& Welfare Committee of Tianjin Medical University.

4) Histological Studies

Tumor tissues were fixed in $4 \%$ paraformaldehyde, embedded in paraffin, sectioned, and then stained using routine immunohistochemistry method with a monoclonal anti-Nerofilament Light chain antibody (NF-L antibody is purchased from Novus Technology: NB300-132).

\section{Results}

1) IHC staining in human body tissue

IHC staing of NF-L was positive in all 10 paraffins of tongue squamous cell carcinoma sections which suggested that newly formed nerves were observed in tumor microenvironment (details in Figure 1).

2) Animal model development

Murine model of tongue squamous cell carcinoma was gained about 1 week 


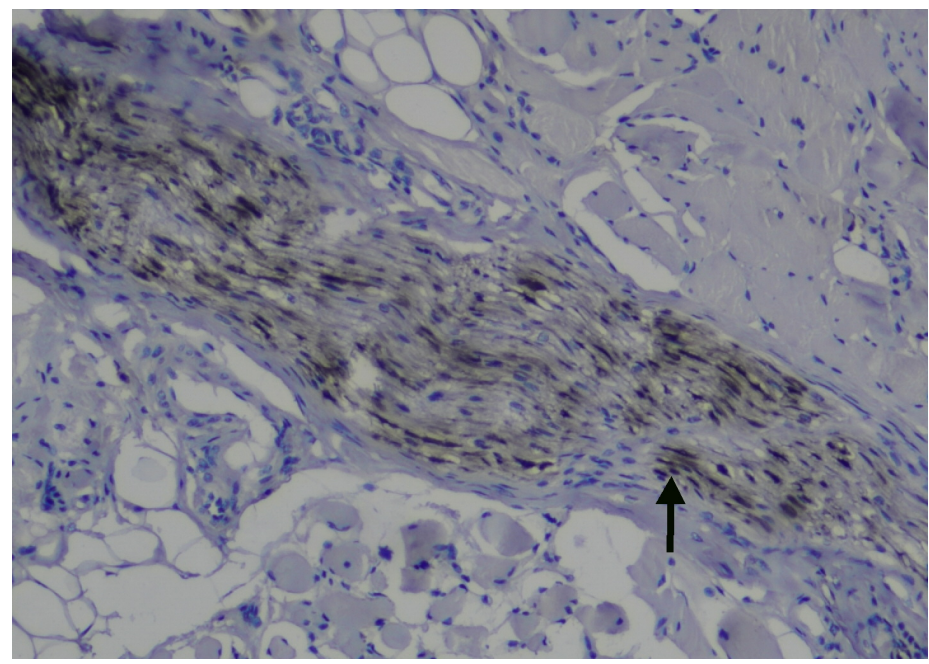

Figure 1. NF-L staining in human tissue. NF-L staining positive indicating new growth of nerves $(\times 100)$.

after cell line injection. Tumor tissues were achieved 2, 4, 6 weeks after cell injection, and fixed in $4 \%$ paraformaldehyde, embedded in paraffin, sectioned.

3) IHC staining in animal model tissue

NF-L staining is positive in the paraffins from animal models even in the murine tissue from 2 weeks indicating that the tongue cancer recruits newly formed nerves in its initiation and development (details in Figure 2(a)). With the growth of tumor in 4 and 6 week, the number of newly formed nerves is increasing (details in Figure 2(b), Figure 2(c)).

\section{Discussion}

One of the most well-known hallmarks of cancer cells is their ability to break away from their original site to invade neighboring tissue and spread to distant body parts [6].

Since the mid-1800s some pioneer scientists described a phenomenon that head and neck cancers exhibited a predilection for growth along nerves namely perineural invasion (PNI) in pathology as they made their way toward the intracranial fossa [7]. Perineural invasion is now well recognized as a significant factor that associated with poor prognosis in several solid malignancies, such as melanoma, prostate cancer, pancreatic cancer, and adenoid cystic carcinoma of the salivary glands [8].

Despite early studies show an impact of denervation in cancer, the role of nerves in cancer initiation and progression has remained unclear [9].

Traditionally, in the relationship between tumor cells and nerves, nerves are passively affected in the process of perineural invasion. Until recently, in a subverted breakthrough article, Claire Magnon has observed for the first time that the infiltration of tumors by growing nerves (tumor neoneurogenesis), and provided evidence that autonomic nerves contribute to prostate cancer development in the mouse model [3]. 


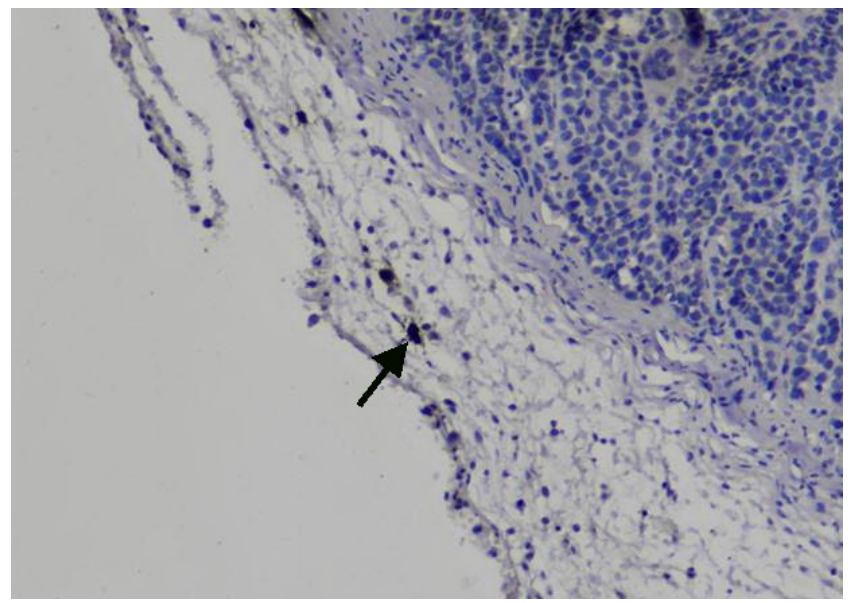

(a)

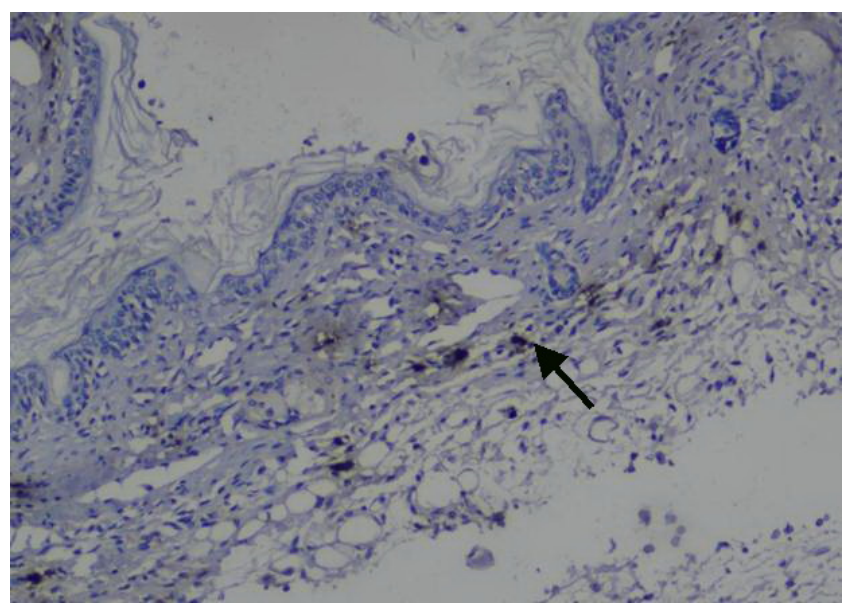

(b)

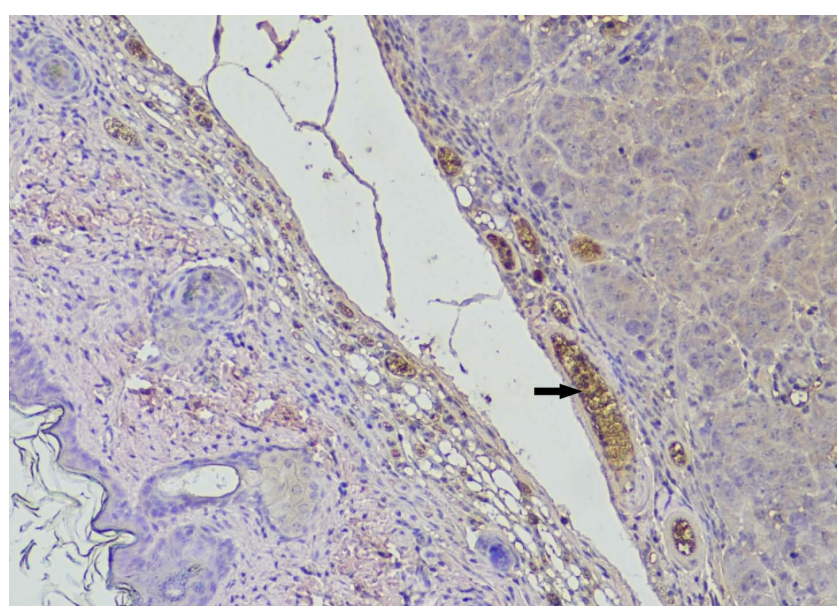

(c)

Figure 2. NF-L staining in murine model. (a) 2 weeks; (b) 4 weeks; (c) 6 weeks. NF-L staining positive indicating new growth of nerves, and the number increasing with tumor growth $(\times 100)$.

The tongue is a strong muscular organ in the mouth which contributes to chewing, swallowing and speech, in addition it is the primary organ of taste in 
the gustatory system. Innervations of the tongue consist of motor fibers, special sensory fibers for taste and general sensory fibers for sensation [10] [11].

In traditional views between the relationship of tongue cancer cells and nerves, nerve fibers are passively affected by cancer cells in tumor environment which leads to numb or local pain and impoverished mobility of the tongue [12] [13].

In our studies, we examined sections of human tongue squamous cell carcinoma tissues by immunohistochemistry. The positive staining of NF-L showed that new growth nerve had been observed in tumor tissue. This analysis revealed that the newly nerve fibers can infiltrate tumor microenvironment actively which suggested that neo-neurogenesis may play an important role in its development.

To explore whether newly nerve fibers could be observed in the initial phase of cancer, an animal experiment was designed. Paraffind sections were achieved from murine grafting. IHC staining of NF-L was positive even in the tissue from 2 weeks and the intensity of nerve fibers increased accompanied with the growth of tumors indicating that neo-neurogenesis may attribute to the pathogenesis and development of tongue squamous cell carcinoma.

More and more studies have discovered that neo-neurogenesis stimulates the initiation and metastasis of several types of cancer. However, the exact underlying mechanism between neo-neurogenesis and cancer has not been clarified. Neurotrophic factors such as nerve growth factor (NGF) secreted from new growth nerves may be a promoter on cancer cell differentiation and proliferation [14] [15]; a recent study by Zahalka shows that new growth adrenergic nerves in prostate cancer microenvironment are critical for activation of an angiogenic switch that fuels exponential tumor growth [16].

For the first time, neo-neurogenesis was detected in the initiation and development of tongue squamous cell carcinoma. The further identification of tumor neo-neurogenesis may provide new insight into the pathogenesis of tongue cancer. From a therapeutic perspective, further studies on the role of neurogenesis in tongue cancer progression may provide new clinical opportunity through anti-neoneurogenesis. Inhibition of new growth of nerve infiltration can be achieved by excision or local injection of neurotoxic agents directly or targeting neurotrophic growth factors and its receptors indirectly.

\section{Conflicts of Interest}

No conflict of interest declared.

\section{References}

[1] Prince, S. and Bailey, B.M. (1999) Squamous Carcinoma of the Tongue: Review. British Journal of Oral and Maxillofacial Surgery, 37, 164-174.

https://doi.org/10.1054/bjom.1999.0031

[2] Kolokythas, A., Park, S., Schlieve, T., Pytynia, K. and Cox, D. (2015) Squamous Cell Carcinoma of the Oral Tongue: Histopathological Parameters Associated with 
Outcome. International Journal of Oral and Maxillofacial Surgery, 44, 1069-1074. https://doi.org/10.1016/j.ijom.2015.01.027

[3] Magnon, C., Hall, S.J., Lin, J., et al. (2013) Autonomic Nerve Development Contributes to Prostate Cancer Progression. Science, 341, Article ID: 1236361. https://doi.org/10.1126/science.1236361

[4] Zhao, C.M., Hayakawa, Y., Kodama, Y., et al. (2014) Denervation Suppresses Gastric Tumorigenesis. Science Translational Medicine, 6, 250ra115. https://doi.org/10.1126/scitranslmed.3009569

[5] Jobling, P., Pundavela, J., Oliveira, S.M., et al. (2015) Nerve-Cancer Cell Cross-talk: A Novel Promoter of Tumor Progression. Cancer Research, 75, 1777-1781. https://doi.org/10.1158/0008-5472.CAN-14-3180

[6] Hanahan, D. and Weinberg, R.A. (2011) Hallmarks of Cancer: The Next Generation. Cell, 144, 646-674. https://doi.org/10.1016/j.cell.2011.02.013

[7] Roh, J., Muelleman, T., Tawfik, O. and Thomas, S.M. (2015) Perineural Growth in Head and Neck Squamous Cell Carcinoma: A Review. Oral Oncology, 51, 16-23. https://doi.org/10.1016/j.oraloncology.2014.10.004

[8] Liebig, C., Ayala, G., Wilks, J.A., et al. (2009) Perineural Invasion in Cancer: A Review of the Literature. Cancer, 115, 3379-3391. https://doi.org/10.1002/cncr.24396

[9] Mancino, M., Ametller, E., Gascón, P. and Almendro, V. (2011) The Neuronal Influence on Tumor Progression. Biochimica et Biophysica Acta (BBA)-Reviews on Cancer, 1816, 105-118. https://doi.org/10.1016/j.bbcan.2011.04.005

[10] Drake, R.L., Vogl, A.W. and Mitchell, A.W.M. (2005) Gray's Anatomy for Students. Churchill Livingstone, Philadelphia, 989-995.

[11] Dudek, R.W. (2014) BRS Embryology. 6th Edition, LWW, Philadelphia.

[12] Binmadi, N.O. and Basile, J.R. (2011) Perineural Invasion in Oral Squamous Cell Carcinoma: A Discussion of Significance and Review of the Literature. Oral Oncology, 47, 1005-1010. https://doi.org/10.1016/j.oraloncology.2011.08.002

[13] Tarsitano, A., Tardio, M.L. and Marchetti, C. (2015) Impact of Perineural Invasion as Independent Prognostic Factor for Local and Regional Failure in Oral Squamous Cell Carcinoma. Oral Surgery Oral Medicine Oral Pathology Oral Radiology, 119, 221-228. https://doi.org/10.1016/j.oooo.2014.10.004

[14] Adriaenssens, E., Vanhecke, E., Saule, P., et al. (2008) Nerve Growth Factor Is a Potential Therapeutic Target in Breast Cancer. Cancer Research, 68, 346-351. https://doi.org/10.1158/0008-5472.CAN-07-1183

[15] Pundavela, J., Demont, Y., Jobling, P., et al. (2014) ProNGF Correlates with Gleason Score and Is a Potential Driver of Nerve Infiltration in Prostate Cancer. The American Journal of Pathology, 184, 3156-3162. https://doi.org/10.1016/j.ajpath.2014.08.009

[16] Zahalka, A.H., Arnal-Estapé, A., Maryanovich, M., et al. (2017) Adrenergic Nerves Activate an Angio-Metabolic Switch in Prostate Cancer. Science, 358, 321-326. https://doi.org/10.1126/science.aah5072 